\title{
The efficacy of alcohol-based hand sanitizers used in a series, modifying the ASTM E2755 method with a shorter hand sanitizer application time
}

Scott Christie ${ }^{1}$, Bobby Sidhu ${ }^{2}$

1 Lead Author, B. Tech Student, School of Health Sciences, British Columbia Institute of Technology, 3700 Willingdon Ave, Burnaby, BC V5G 3H2

2 Supervisor, School of Health Sciences, British Columbia Institute of Technology, 3700 Willingdon Ave, Burnaby, BC V5G 3H2

\begin{abstract}
Background: Alcohol-based hand sanitizers are becoming increasingly common in healthcare settings in an effort to control communicable bacteria, viruses and fungi of health significance. Much research has been done on the effectiveness of alcohol-based hand sanitizers but few studies look at the effectiveness when combined with more typical usage, such as varying application times and amounts.

Methods: We looked at the efficacy of the microbial killing power of an alcohol-based hand sanitizer when used in a series of 5 applications. ASTM E2755 was used with the modification of a shorter application time (8 seconds from the recommended 30 seconds) of the sanitizer to better reflect actual healthcare worker usage.

Results: We found an increase in the amount of indicator bacteria on the gloved hands of the subjects after repeated applications. However the increase was not significant enough in that a 2-log reduction of indicator bacteria was still achieved. Using a One Sample T-Test we found a very low probability value $(<0.00000)$, indicating that the results were statistically significant.

Conclusions: There is an increase of bacteria on gloved hands after repeated use of alcohol-based hand sanitizers. The results show a decrease in their effectiveness, most likely due to a build up of various nonalcohol components in hand sanitizers. However even with the 8 second application time there was still a greater than two log reduction even after 5 serial contaminations and applications. This cautiously shows that there is significantly less danger posed by more common shorter application times than originally thought. These findings have a potential impact on hand hygiene education as other factors, such as frequency or sanitizer amounts can be safely emphasized over application times.
\end{abstract}

Key Words: alcohol hand sanitizer, hand hygiene, ASTM E2755, application time, nosocomial infections

\section{Introduction}

Healthcare associated infections also know as nosocomial infections are a significant problem for many developing and developed countries. Patients that contract nosocomial infections can have prolonged hospital visits, long-term disabilities, incur greater healthcare costs, increase the amount of antibiotic resistant flora and even increased mortality and morbidity (Plowman et al., 2001). Nosocomial infections are a significant problem with an unknown incidence and prevalence, as it is very difficult to directly associate an newly acquired infection with a patient's environment. The diagnosis of such an infection requires multiple criteria, sampling, surveillance and not just a single diagnostic test.

In developed countries nosocomial infections can affect 5-15\% of all patients and up to $37 \%$ of patients admitted to intensive care units (ICU) (WHO, 2005). In the US the incidence rate was $4.5 \%$ in 2002, with around 1.7 million patients affected (Klevens et al., 2002).
At many hospitals, the use of alcohol-based hand sanitizers is encouraged as a method to promote hand hygiene, a critical issue among front-line healthcare workers (Wilfinger, 2011).

Many front line staff responsible for patient care use hand sanitizers as they are much quicker to use than washing hands with soap and water. For example, a dialysis technician will be responsible for multiple machines that they must set-up, take down and disinfect in a relatively short time, during a patient turn-over period. Consequently this can result in the repeated application of an alcohol-based hand sanitizer without hand washing in an effort to ensure patients receive their treatment in a timely manner while still complying with hand hygiene protocols. Similarly in a Dialysis ward, nursing staff also must also attend to multiple patients within a relatively short time period, making alcohol-based hand sanitizers a popular choice due to their convenience (Christie, personal communication, 2013).

Although a number of studies have been 
done on the efficacy of alcohol-based hand sanitizers, mainly using the American Society for Testing and Materials standardized methods, they do not attempt to simulate normal working conditions, mainly a more realistic application time for alcohol-based hand sanitizers. This study aims to fill that gap by supplementing the standard method with a reduction of the application time of the sanitizer to better mimic conditions experienced by front-line healthcare workers. The information provided by this study will add critical information to the knowledge base on alcohol-based hand sanitizers. The results of the study will also aid in determining if there is a danger posed by the incorrect use of hand sanitizers.

\section{Literature Review}

Although the antimicrobial properties of alcohol have been well documented the first major guidelines for the use of alcohol-based hand sanitizers was developed by the Association for Professionals in Infection Control (APIC) in 1995 (Larson, 1995). It staunchly supported the use of alcohol-based hand sanitizers in clinical healthcare settings. In 1996 the Healthcare Infection Control Practices Advisory Committee (HICPAC) recommended that antimicrobial soap or a waterless antiseptic agent, such as an alcohol hand rub, be used by healthcare workers after attending to patients with multidrug resistant pathogens such as vancomycin-resistant enterococci (VRE) and methicillin-resistant Staphylococcus aureus (MRSA) (Garner, 1995).

The human skin is composed of three main layers, the epidermis $(50-100 \mu \mathrm{m})$, dermis $(1-2 \mathrm{~mm})$ and the hypodermis $(1-2 \mathrm{~mm})$. One of the main functions of the skin is to retain moisture and to prevent the entrance of pathogens. When the skin experiences damage in the form of chemical irritation or removal of lipids by non-polar solvents (such as soap) it reacts by increasing keratinocyte proliferation and differentiation. The increase in keratinocytes cause the formation of callouses. These changes directly affect the ability of the skin to uptake nutrients, protect against water loss and synthesis of new proteins and lipids. Thus as necessary as hand washing is, it is detrimental as it can indirectly contribute to pathogen survival and transmission (WHO, 2009).

The human skin contains a number of bacterial flora, both transient (acquired) and resident (local). Total bacterial counts on the hands of medical personnel have ranged from 3.9 x $10^{4}$ to $4.6 \times 10^{6}$ cells (Larson et al., 1998). Transient flora, usually colonize the more outer, superficial layers of the skin and thus can be more easily removed by hand hygiene. Resident flora, which colonize deeper layers are more resistant to removal by hand hygiene. Resident flora are less likely to be associated with human infections, however it can still cause infections when dealing with unprotected areas such as sterile body cavities, eyes, broken-skin or other mucous membranes (Lark et al., 2001). Resident flora serve two important and helpful functions, competition of nutrients and microbial antagonism against transient flora. Staphylococcus epidermis is the dominant resident species, others include $S$. hominis and coryneform bacteria such as propionibacteria, corynebacteria, dermobbacteria and mircococci (Rayan, 1987). Fungi are also of a concern with the most common resident flora genus being Pityrosporum spp. Hands of health care workers can become temporarily colonized by pathogenic flora such as Staphylococcus aureus and in some cases may even persist. One study showed that strains of Escherichia coli and Klebsiella spp. had a 50\% reduction after 6 and 2 minutes respectively (Fryklund, 1995). Another study showed that Enterococcus faecalis and E. faecium survived for 60 minutes on gloved and ungloved hands (Noskin et al., 1995). Although the amount of transient and resident bacteria varies greatly from person to person, it is generally found to be relatively constant for each individual (Sprunt et al., 1973).

Hand hygiene, especially in a healthcare setting is vital as they can serve as a mode of transmission. Organisms that are present on the patient or that have been shed onto objects can be transferred to the healthcare worker's hands. To ensure transmission, the organisms must be able to survive several minutes on the hands of the worker. Inadequate, or omitted, hand washing or hand antisepsis must also occur, allowing for the survival of the organism. Finally these contaminated hands must come into contact with the patient or onto objects that will come into contact with the patient for the organism to be transmitted. The majority of studies show that a two-log reduction in the number of viable cells is the threshold to achieve when trying to prevent transmission (Pittet et al, 2006). The Food and Drug Administration requires a 2 log reduction on the first application of any hand sanitizer and Health Canada requires a 3 log reduction (FDA, 2004), (Health Canada, 2009). 
Currently there are several methods to examine the effectiveness of hand hygiene products. The most relevant method being ASTM E2755 - 10, Standard Test Method for Determining the Bacteria-Eliminating Effectiveness of Hand Sanitizer Formulations Using Hands of Adults.

These standard evaluation methods call for $3 \mathrm{~mL}$ of alcohol rub to be applied and rubbed into the hands for 30 seconds. As noted by the United States Center for Disease Control this type of protocol does not reflect actual usage patterns among healthcare workers (CDC, 2002). The Fraser Health orientation manual given to new employees has the guideline of a maximum 5 times use of alcohol-based hand sanitizer in a row, assuming the hands were not visibly soiled, before hand washing with a detergent is required. Their directions for using alcohol hand rubs say for the products to be rubbed into the hands until they are dry, and this may not reflect actual usage patterns (FHA, 2013).

A number of factors can affect the effectiveness of alcohol-based hand sanitizers, this can include: type of alcohol, concentration, contact time, volume, viscosity and amount of water on the hands prior to application. The majority of alcohol-based sanitizers contain either ethanol, isopropanol or n-propanol or a combination of these products. For the most part there are no significant differences in the disinfection capability of ethanol compared to isopropanol, except in the area of viruses in which ethanol has greater effect (Kampf \& Kramer, 2004). The ability of alcohol to destroy microorganisms comes from its intrinsic ability to denature proteins. Alcohol solutions that are around $60-80 \%$ have the highest ability to denature, with higher concentrations actually having less potency (Larson \& Morton, 1991). This counter intuitive fact is due to proteins being less susceptible to denaturation in the absence of water. The alcohol content of any product should be expressed as a percentage of weight (mass/mass) or a percentage of volume (volume/volume), as it is not altered by factors such as temperature. The volume of sanitizer used is also important, with the recommended amount being $1.5 \mathrm{~mL}$ for both hands. Smaller volumes can provide insufficient coverage and thus insufficient sanitization. Alcohol has good germicidal activity against both gram-positive and gram-negative bacteria, and even those that are drug resistant such as MRSA and VRE, as well as Mycobacterium tuberculosis and fungi (Price, 1996). However where alcohol fails as a disinfectant is in the realm of bacterial spores, protozoan cysts, and against some non-enveloped viruses.

There are a number of sanitizers available on the market, but this study will focus on Isagel. Fraser Health Authority primarily uses the hand sanitizer Isagel, as manufactured by the Coloplast Corporation. The main medical ingredient of Isagel being 60\% Alcohol-Ethyl denatured alcohol, it also has added moisturizers, surfactants and thickeners in the form of carbomer, glycereth-26, PEG-12, triethanolamine and purified water (Coloplast, 2012).

\section{Methods}

This study followed closely the ASTM E2755 methods for testing the efficacy of the alcohol hand sanitizer. However, the test product application procedure has been altered from the normal ASTM E2755 standard method, to better simulate real working conditions experience by healthcare workers. The standard method calls for $1.5 \mathrm{~mL}$ to be dispensed and rubbed on the hands and until dry, usually around 30 seconds. (ASTM E2755, 2010) The instructions for the application of alcohol hand sanitizers as released by the British Columbia Ministry of Health also for the sanitizer to be rubbed into the hands until dry (BC Min. of Health, 2012).

However, most healthcare worker's application times are much shorter. As noted by separate studies conducted by researchers Rotter and Pittet, application times for healthcare workers typically range from 8-12 seconds (Rotter, 1999), (Pittet et al., 2000). Also of note, participants of a infection control class, a population consisting of highly motivated healthcare personnel, 57\% (1783/3105) of participants fell within the 16-29 seconds application time (Hautemaniere et al., 2010). Thus in an effort to simulate more realistic application times, an 8 second, "worst-case" application time will be used.

Bacterial recovery was done using the "glove juice technique". Within a minute of the application of the alcohol hand sanitizer attach a glove to the subject's gloved hands. Add $75 \mathrm{~mL}$ of the Sampling Solution, consisting of Trition $\mathrm{X}-100$ combined with a buffering solution, to each glove and secure the gloves at the wrist with a tourniquet. Then massage the surfaces of the subjects gloved hands and fingers thoroughly for 1 minute. After, retrieve $5 \mathrm{~mL}$ sample of the sampling solution from each glove. Dilute the sample as needed and plate within 30 minutes 
after retrieval. (ASTM E2755, 2010).

The study is conducted via the following steps: 1.) Preparation of Test Bacteria, 2.) Testing Admission, 3.) Cleansing Wash, 4.) Hand Contamination, 5.) Bacterial Recovery, 6.) Hand Contamination, 7.) Test Product
Applications with Steps 6 \& 7 repeated 5 times in a series, 8.) Bacterial Recovery, 9.) Enumeration of Bacteria, 10.) Hand Decontamination. The steps 2-10, including the repetition of $6 \& 7$, constitute one "run", and a total of 20 "runs" are conducted.

\section{Results}

\begin{tabular}{|l|l|l|}
\hline \multicolumn{1}{|c|}{ Initial Culture CFU's } & Baseline CFU's & \multicolumn{1}{c|}{ Post-Treatment CFU's } \\
\hline 1000000000 & 0 & 1750 \\
\hline 1000000000 & 0 & 960 \\
\hline 1000000000 & 0 & 820 \\
\hline 1000000000 & 0 & 1320 \\
\hline 1000000000 & 0 & 1340 \\
\hline 1000000000 & 0 & 1560 \\
\hline 1000000000 & 0 & 1080 \\
\hline 1000000000 & 0 & 770 \\
\hline 1000000000 & 0 & 1620 \\
\hline 1000000000 & 0 & 1550 \\
\hline 1000000000 & 0 & 840 \\
\hline 1000000000 & 0 & 910 \\
\hline 1000000000 & 0 & 1030 \\
\hline 1000000000 & 0 & 1270 \\
\hline 1000000000 & 0 & 1300 \\
\hline 1000000000 & 0 & 1180 \\
\hline 1000000000 & 0 & 1130 \\
\hline 1000000000 & 0 & 2540 \\
\hline 1000000000 & 0 & 1460 \\
\hline & & \\
\hline & 0 & 0 \\
\hline
\end{tabular}




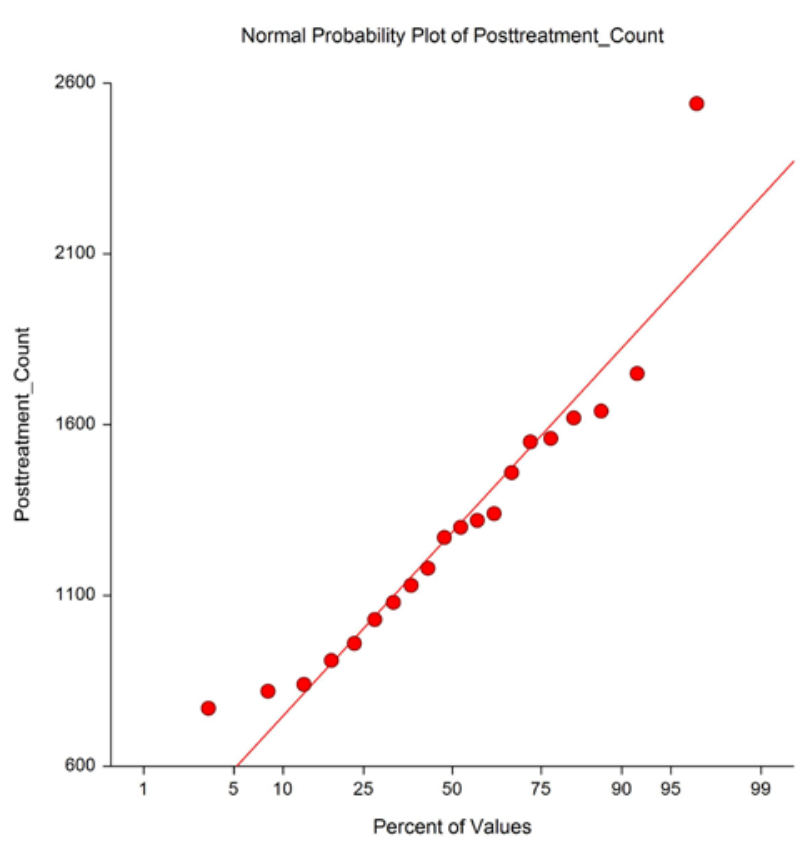

As we can see from the results it has a very low probability value $(0.00000)$, indicating that it has a highly statistically significant result and that we can reject the null hypothesis. This data shows there to be a statistically significant difference between the initial culture CFU's and the recovered post treatment CFU's. This means that it is very unlikely these differences occurred by random chance. Given the median CFU's (1285), mean CFU's (1303.5) or even the highest value of CFU's (2540), we can see how this is still a large reduction from the initial culture CFU's of $10^{9}$. The $2 \log$ or $3 \log$ reduction as required and recommended has very much been achieved.

Looking at the tests of assumption, we can see that the data is skewed and has one potential outlier (2540 CFU's) to it, meaning that a non-parametric test is suitable. The power of this study, is extremely high (1.00000) at both at 0.05 and 0.01 . This implies a strong trend that the null hypothesis is correctly rejected.

\section{Discussion}

This novel, standard method based study has provided new data in the realm of hand hygiene and on the nature of alcohol-based hand sanitizers in particular. The use of the American Society for Testing and Materials standard

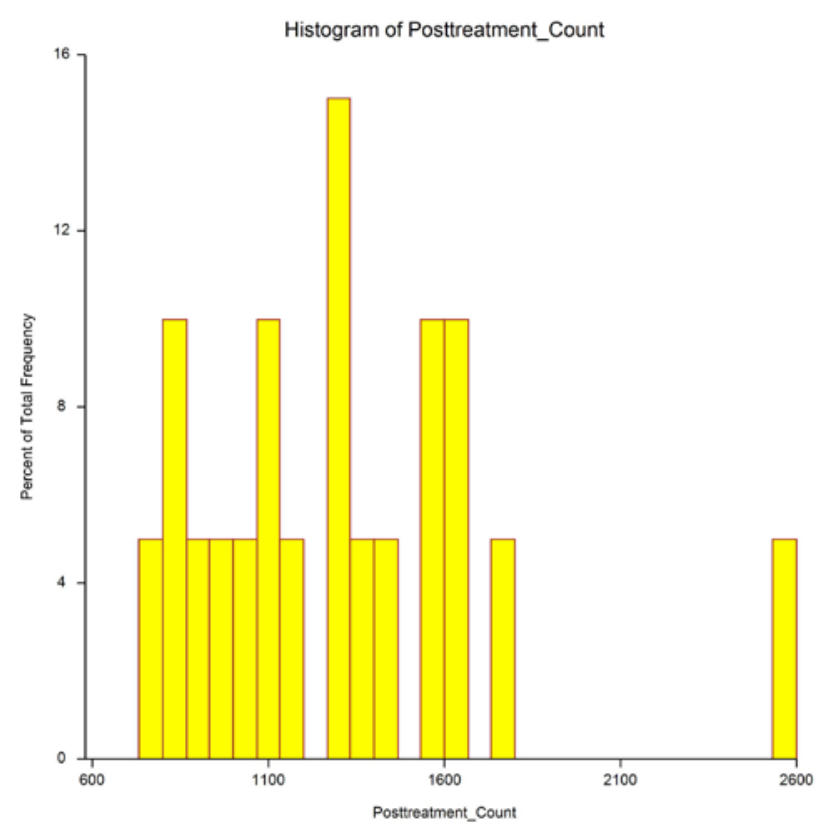

method E2755 combined with a slight modification to better reflect more realistic conditions has given new insight into the nature of alcohol-based hand sanitizers. Through the standard method, we have shown that there is still an increase in the amount of bacteria with short, serial applications of hand sanitizer, though not as great as feared. The end increase in bacterial load is roughly in the thousands, but still a far amount away from the initial $10^{9}$ CFU's. Although the amount of bacteria that remains is certainly still viable and potentially infectious, the amount of reduction is sufficient to stop the majority of transmission. Total sterility under normal, non-surgical hospital ward working conditions, with only the use of an alcohol-hand sanitizer, is nearly impossible. Through this study it has been also shown that an appropriate, even application of alcohol-based hand sanitizer is possible and easily achieved within 8 seconds. This is evidenced in the lack of viable bacteria in the initial baseline recovery plates, all which had a total of 0 CFU's.

As evidenced by the results, there is an increase in the bacterial load after repeated serial applications of the alcohol-based hand sanitizer. This is most likely caused by the build up of various non-alcohol components of the hand sanitizer. This may include the various added moisturizers, surfactants and thickeners in the form of carbomer, glycereth-26, PEG-12 and 
triethanolamine (Coloplast, 2012). Though it is hard to say what the actual composition of the build up is, without further detailed chemical and microbiological analysis. During the applications of the indicator bacteria and subsequent hand sanitizer application, it was noticed, in particular that the indicator bacteria and sanitizer would combine to form small, numerous, beige-colored, glue-like textured, spheres. This was especially noticed on the fifth and last application, with many spheres being formed. This is in stark contrast to the initial application when virtually none of these spheres were present. This may be due to the tryptic soy broth, from which the indicator bacteria was delivered, combining with the alcohol hand sanitizer in an unknown reaction. However it is most likely due to the moisture repellent nature of the latex gloves. The nature of the latex gloves are in stark contrast to the inherent properties of live human skin, which are much more absorptive of the various components of the alcohol-based hand sanitizer. The exact properties and components of these spheres however are unknown until further research is conducted. This build up or spheres is most likely the reason for the Fraser Health Authority guidelines on 5 serial applications being the maximum amount, before hand washing should occur. It is conceivable that the spheres provided the indicator bacteria physical protection from the inactivation ability of subsequent hand sanitizer applications.

The lack of viable bacteria in the initial baseline recovery plates shows two things. Firstly, that the hand sanitizer does indeed work as advertised, achieving an impressive 9 log reduction and the elimination of all viable indicator bacteria on the first contamination and application cycle. Secondly, it also shows that appropriate coverage, that is to say effective use of the hand sanitizer, is viably achieved even with the limited 8 second application time. This is contrary to the advised usage of the hand sanitizer, with the manufacturer Coloplast recommending a 15 second application time and Health Canada recommending a 15-30 second application time (Coloplast, 2012) (Health Canada, 2009). Fraser Health recommends a 1520 second application time, while Vancouver Coastal health recommends the sanitizer to be applied and rubbed until the hands are dry. The experiment met none of the above criteria with its 8 second application time, which also left the gloves wet with non-evaporated alcohol hand sanitizer. Again it is important to note that the use of latex gloves, which are specifically designed to repel moisture do not have the same absorptive qualities as human skin. This in part accounts for the wetness or leftover hand sanitizer on the gloves. Anecdotally the 8 second application time of hand sanitizer on live human skin leaves hands fairly, but not completely dry. This indicates that the Vancouver Coastal Health suggested application could be partially fulfilled if this experiment was repeated using human skin instead of gloves even with the limited application time. Also this reduction of viable bacteria with the limited application time correlates with previous research. As noted previously by researchers Rotter and Pittet in separate studies, the shorter 8 second application time is the norm for many health care workers (Rotter, 1999), (Pittet et al., 2000). Anecdotally, this researcher has also seen an 8 second application time or shorter to be the norm at the Royal Columbian Hospital (RCH) Hemodialysis Ward, and the norm in other units at RCH (Christie, personal communication, 2013). It reasons that if the 8 second application time were to produce more disastrous results, meaning an insufficient inactivation of viable bacteria, viruses or fungi, no doubt this would show in increases of nosocomial infections rates following the introduction of alcohol-based hand sanitizers in hospitals as a viable alternative to traditional hand washing with soap and water. However this is clearly not the case as nosocomial rates have decreased in the majority hospital wards upon the introduction of hand sanitizers (Pessoa-Silva et al., 2004) (Strausbaugh et al., 2006).

Hand hygiene is considered as one of the best and most basic measures in infection control to prevent the transmission of nosocomial pathogens. As shown in many previous studies hand hygiene, no matter what the form it is in, whether soap or alcohol, has a significant impact on the risk of nosocomial infection rates (Plowman, 2001) (WHO, 2009). There have been many studies on the effectiveness of alcohol-based hand sanitizers when used as per instructed, and some on the presence of bacteria on the hands of healthcare workers. However, there has been no peer-reviewed published research of quantitative studies on the effectiveness of alcohol-based hand sanitizers used with a shorter application time that this researcher could find. Also as noted by the US CDC, there seems to be very few studies that reflect actual working usage of the hand sanitizers by health care workers (US CDC, 
2002). Overall, there seems to be no significant danger in this regard of the non-adherence to usage guidelines of alcohol-based hand sanitizer.

\section{Recommendations}

A significant finding, other than alcohol-based hand sanitizers are extremely effective on the first application, is that a shorter application time does not have as significant effect on the reduction of bacteria as possibly thought. This has implications particularly in the realm of hand hygiene education and auditing. These findings suggest that it is more appropriate and effective to focus on other components of hand hygiene such as proper coverage, or proper amounts rather than duration of application. It also tentatively suggests that outbreaks of communicable bacteria are not caused by shorter application times, but other factors.

\section{Limitations}

This study provides some indication of the effective of shorter application times, but its results should be interpreted with caution. A major limitation of this study is the use of gloved hands in the place of actual live human skin. Although an effort was made to ensure that the gloves used were sanitized and properly inoculated, the nitrile gloves are physically and chemically different than skin. The gloves do not have the same texture, $\mathrm{pH}, \mathrm{a}_{\mathrm{w}}$, cutaneous layering, and adsorption properties as human skin does. Consequently this would likely affect the results. This was done to meet the requirements of British Columbia Institute of Technology ethics review board requirements.

Another limitation was deviating away from the standard method ASTM E2755 in terms of the indicator culture used. Although the indicator culture was still $S$. marcescens, the isolate recommended for use is American Type Culture Collection (ATCC) 6538. Unfortunately this isolate was not financially feasible to be obtained and another isolate was used instead. This also may have an impact on results, though most likely not a significant one.

Another limitation of the study is with the small sample size collected. In this study only 20 results were collected, and all of those results were from the researcher. With a sample size this small, the precision is not as great as a study with a larger sample size would be. Also the samples obtained reflect the application technique of only one person. If there were more subjects the range of the data could potentially be wider.

\section{Future Research Suggestions}

One expansion could be done using different types of alcohol-based hand sanitizers, such as foams versus gels. The various brands from each company could also be tested, and from that information hopefully what ingredients cause a build-up residue on the hands could be identified. Another aspect would be to alter the application amounts to be more typical of the average usage. A final study could be done altering both application times and amounts to best reflect actual usage, and to determine if this poses any health concern. In addition any of the previous research suggestions could be done using either fungi or viruses as their indicators rather than bacteria as they may be affected differently.

\section{Conclusions}

The use of a shorter application time does not appear to have a significantly detrimental effect on the ability of the alcoholbased hand sanitizers to kill viable bacteria. The recovered bacteria after repeated applications indicate a decrease in the efficacy of the hand sanitizer. However this decrease in efficacy has led to only a modest increase in bacteria at the end of 5 contaminations and application cycles. This increase in bacteria is still far below the required 2 or 3 log reduction.

Proper use and education about alcoholbased hand sanitizers is key to preventing nosocomial infections due to unclean hands and protecting the public health. Key issues include the appropriateness of products, use of those products and education. Ultimately a lack of knowledge in this area can lead to severe consequences for the health of patients.

\section{Acknowledgements}

I would like to thanks the efforts of the Environmental Health faculty at BCIT for giving me the education, skills and support necessary for this study to be done. I would also like to thank Bobby Sidhu, my supervisor for this project for his helpful comments in improving this paper and particularly for his knowledge of statistics which helped to validate the results. I 
would also like to thank Ken Keilbart for allowing me to use the BCIT Food Technology lab to conduct the necessary microbiological laboratory work.

\section{Competing Interests}

The authors declare that they have no competing interests.

\section{References}

Christie, S., Sidhu, B. (2014) The efficacy of alcohol-based hand sanitizers used in a series, modifying the ASTM E2755 method with a shorter hand sanitizer application time. Journal of Environmental Health of British Columbia Institute of Technology. Spring 2014. Burnaby, British Columbia Canada.

Plowman, R., Graves, N., Griffin, M. A. S., Roberts, J. A., Swan, A. V., Cookson, B., \& Taylor, L. (2001). The rate and cost of hospitalacquired infections occurring in patients admitted to selected specialties of a district general hospital in England and the national burden imposed. Journal of Hospital Infection, 47(3),

198-209.

World Alliance for Patient Safety. The Global Patient Safety Challenge 2005-2006, Clean Care is Safer Care. Geneva, World Health Organization. "http://www.who.int/gpsc/en/” accessed December 2008.

Klevens R et al. (2002) Estimating health careassociated infections and deaths in U.S. Hospitals. Public Health Report. 122:160-166

Wilfinger C. (2011) Innovative Initiatives to Promote Hand Hygiene. American Journal of Infection Control. 39:5.

Larson EL, APIC Guidelines Committee. (1995) APIC guideline for handwashing and hand antisepsis in health care settings. American Journal of Infection Control. 23:251-69.

Garner JS, Hospital Infection Control Practices Advisory Committee. (1996) Guideline for isolation precautions in hospitals. Infection Control Hospital Epidemiology. 17:53-80.

World Health Organization. (2009) WHO Guidelines on Hand Hygiene in Health Care.
Geneva, Switzerland. Retrieved from: http://whqlibdoc.who.int/publications/2009/9789 241597906_eng.pdf?ua=1

Larson EL, Norton Hughes CA, Pyrak JD, Sparks SM, Cagatay EU, Bartkus JM. (1998) Changes in bacterial flora associated with skin damage on hands of health care personnel. American Journal of Infection Control. 26:51321.

Lark RL et al. (2001) An outbreak of coagulasenegative staphylococcal surgical-site infections following aortic valve replacement. Infection Control and Hospital Epidemiology. 22:618-623

Rayan GM, Flournoy DJ. (1987) Microbiologic flora of human fingernails. Journal of Hand Surgery (America) 12:605-607.

Fryklund B, Tullus K, Burman LG. (1995) Survival on skin and surfaces of epidemic and non-epidemic strains of Enterobacteria from neonatal special care units. Journal of Hospital Infection. 29:201-208

Noskin GA et al. (1995) Recovery of vancomycin-resistant Enterococci on fingertips and environmental surfaces. Infection Control and Hospital Epidemiology. 16:577-581.

Sprunt K, Redman W, Leidy G. (1973) Antibacterial effectiveness of routine hand washing. Pediatrics. 52:264-71.

Pittet, D., Allegranzi, B., Sax, H., Dharan, S., Pessoa-Silva, C. L., Donaldson, L., \& Boyce, J. M. (2006). Evidence-based model for hand transmission during patient care and the role of improved practices. The Lancet infectious diseases,6(10), 641-652.

Food and Drug Administration. Tentative final monograph for healthcare antiseptic drug products; proposed rule. Federal Register 59, 31441-31450 (2004).

Health Canada. (2009) Guidance Document: Human-Use Antiseptic Drugs. Health Products and Food Branch, Ministry of Health.

Centers for Disease Control and Prevention. (2002) Guideline for Hand Hygiene in Health Care Settings: Recommendations of the Healthcare Infection Control Practices Advisory Committee and the HICPAC/SHEA/APIC/IDSA 
Hand Hygiene Task Force. MMWR. 51:16.

Fraser Health Authority. (2013). Orientation Manual for Dialysis Technicians. Surrey, British Columbia: Fraser Health.

Kampf G, McDonald C, Ostermeyer C. (2005) Bacterial in-use contamination of an alcoholbased hand rub under accelerated test conditions. Journal of Hospital Infection. 59:271272.

Larson EL, Morton HE. (1991) Alcohols. In: Block SS, ed. Disinfection, sterilization and preservation, 4th ed. Philadelphia, PA, Lea \& Febiger. 191-203.

Price PB. (1996) New studies in surgical bacteriology and surgical technique. Journal of American Medicine. 111:1993-1996.

Coloplast Corporation. (2012). Material Safety Data Sheet: Isagel. Mississauga, Ontario: Coloplast Corporation.

American Society for Testing and Materials. (2010) ASTM E2755-10,Standard Test Method for Determining the Bacteria-Eliminating Effectiveness of Hand Sanitizer Formulations Using Hands of Adults. West Conshohocken, PA: American Society for Testing and Materials.

Rotter, M.L. (1999) Hand washing and hand disinfection. CG Mayhall (Ed.), Hospital epidemiology and infection control, 2nd Ed. Lippincott Williams and Wilkins, Philadelphia. 1339-1355.

Pittet D., Hugonner S., Harbarth S. (2000) Effectiveness of a hospital-wide programme to improve compliance with hand hygiene. Lancet, 354, pp. 1307-1312.

Hautemaniere A., Cunat L., Diguio N., Vernier N., Schall C., Daval M.C., Ambrogi V., Tousseul S., Hunter P.R., Hartemann P. (2010) Factors determining poor practice in alcoholic gel hand $r u b$ technique in hospital workers. Journal of Infection and Public Health. 3:1, 25-34.

Pessoa-Silva CL et al. (2004) Dynamics of bacterial hand contamination during routine neonatal care. Infection Control and Hospital Epidemiology. 25:192-197.

Strausbaugh, L. J., Siegel, J. D., \& Weinstein, R.
A. (2006). Preventing transmission of multidrugresistant bacteria in health care settings: a tale of two guidelines. Clinical infectious diseases, 42(6), 828-835. 